\title{
High-resolution Arteriography of the Major Peripheral Vessels
}

\author{
LARS BJÖRK and TAMAS SANDOR
}

From the Department of Radiology, Harvard Medical School and Peter Bent Brigham Hospital, Boston, Massachusetts, USA

\begin{abstract}
In experimental studies using a $0.1 \mathrm{~mm}$ focal spot tube, rare-earth screens, Cronex 4 film and diluted contrast agent $(120 \mathrm{mgI} / \mathrm{ml}), 6 \times$ magnification showed detail in the walls of major arteries that could not be identified with standard radiography or $3 \times$ magnification. The method is applicable to studies of arteriosclerotic changes in the wall of the femoral artery in patients.
\end{abstract}

\section{INTRODUCTION}

High-degree magnification radiography can enhance the visualization of small image details and can even delineate small vessels not visible on the original radiograph (1). Although magnification has little effect on larger images, such as those of major arteries, it can significantly improve delineation of the walls of these arteries. We are currently developing an angiographic method for the study of early small arteriosclerotic changes in large vessels. Such a technique will be useful in man for early diagnosis and objective assessment of the effects of dietary and/or drug treatment for arteriosclerosis.

The experiments described here dealt with magnification of the femoral artery in dogs and autopsy specimens of human superficial femoral arteries.

\section{THEORETICAL CONSIDERATIONS AND EXPERIMENTAL PROCEDURES}

The efficiency of early diagnosis of arteriosclerosis depends on how well the walls of the artery can be visualized. In this context, improved image quality means an image that has better contrast and higher resolution than can be obtained with conventional techniques. Such improvement was recently assessed with regard to the contrast of small blood-vessel images. Image contrast was calculated in terms of magnification by using computer simulation technique (1); the results are shown in Fig. 1. The curves indicate that a significant increase in contrast occurs when the effective focal-spot size is smaller than $0.2 \mathrm{~mm}$ and magnification is about $6 \times$ (provided that a par-speed film-screen combination or an equivalent line spread function is used). These results have been experimentally verified (1) and were applied in the present study.

\section{EXPERIMENTS IN DOGS}

\section{Material and Methods}

Six mongrel dogs weighing 20 to $25 \mathrm{~kg}$ were anesthetized with an intravenous injection of sodium barbiturate. They were intubated but no artificial respiration was used. A catheter was introduced into the femoral artery on one side and manipulated via the aortic bifurcation into the distal femoral artery on the other side. This artery was exposed at the groin to permit subsequent occlusion of the artery around the catheter.

An X-ray tube with a $0.1-\mathrm{mm}$ focal spot was used for taking all magnification arteriograms. The design of the tube was such that it had an inherent current flow of $\mathbf{3 0}$ $\mathrm{mA}$; the kilovoltage had a range of 50 to $100 \mathrm{kV}$ with 10 $\mathrm{kV}$ increments.

Single exposures were obtained of the femoral artery at the mid-thigh. Exposure factors were 60 to $80 \mathrm{kV}$ and 30 $\mathrm{mA}$; exposure time varied from 0.2 to $1.2 \mathrm{sec}$. Magnification of 3 to $10 \times$ was used. Special rare-earth screens (a mixture of terbium-activated yttrium and gadolinium oxysulfide) with high definition were used in combination with Cronex 4 film. These screens had an 8 to 10 line pairs $/ \mathrm{mm}$ resolution measured with a line test pattern at $40 \mathrm{kV}$.

The injection of contrast medium was prolonged to approach homogeneous filling of the arterial system during the long exposure times used in this experiment. Injections were made either by hand or by an automatic injector. In both instances the rate of injection was adjusted to permit complete replacement of blood flow with contrast medium. The contrast media were Renografin 60 with an iodine content of $290 \mathrm{mg} / \mathrm{ml}$ and Renografin 60 


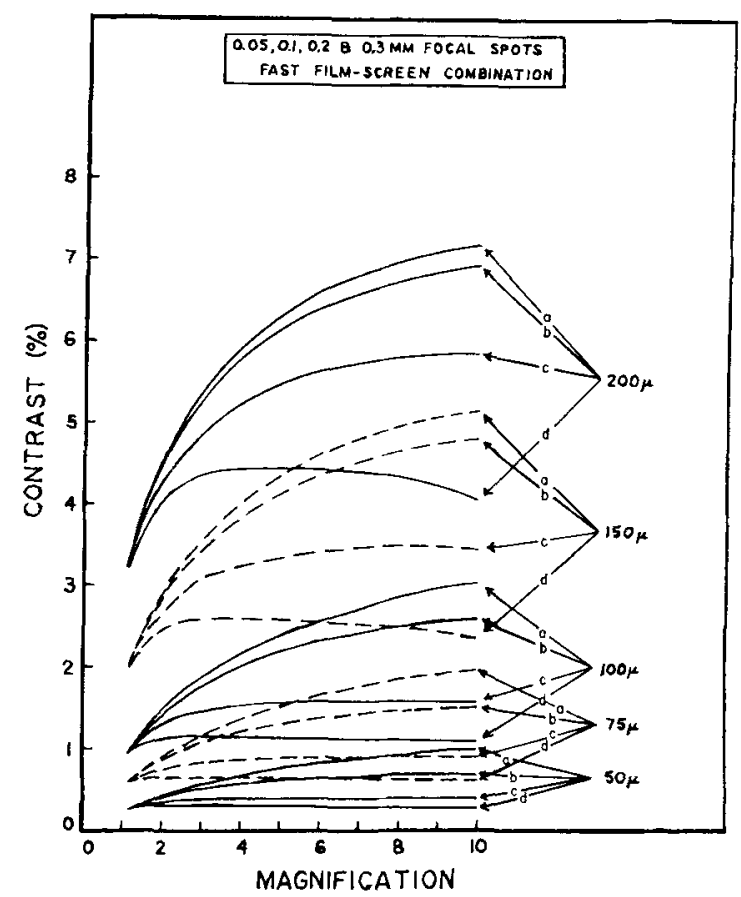

Fig. I. Contrast calculated in terms of magnification for $50,75,100,150$ and $200 \mu \mathrm{m}$ blood vessel images. The dimensions of the focal spots are $0.05,0.1,0.2$ and 0.3 $\mathrm{mm}$. The line spread function of Kodak Blue Brand film and Radelin TF-2 screens were used in the computations.

diluted with distilled water to $70,100,120$ and $145 \mathrm{mgI} / \mathrm{mI}$. In addition, 20 to $30 \mathrm{ml}$ of air using high injection rate was injected after filling the catheter with Renografin 60 . This created a coating of contrast medium on the surfaces of the walls of the arteries distended by the air. Before the injection of air either alone or for the double contrast effect, the femoral artery was occluded by tying a cotton band around the catheter in the groin.

\section{Results}

The best results with regard to definition and contrast were obtained using $70 \mathrm{kV}$ for the exposures. Images of high contrast could be obtained regularly at this $\mathrm{kV}$ value. At the same time the transparency of the images was satisfactory to permit evaluation of wall definition in larger vessels and even evaluation of vessels crossing each other. Exposures were also taken at $6 \times$ with High Plus screens and Cronex 4 film. It was found that the image quality with rare-earth screens was significantly better than that obtained by High Plus and Cronex 4 film-screen combination.

Renografin 60 with an iodine content of 290 $\mathrm{mg} / \mathrm{ml}$ was too concentrated to delineate the fine details of larger vessel walls. Renografin diluted to $120 \mathrm{mg} / \mathrm{ml}$ gave the best results (Fig. 2). The problem of oversaturation of the blood-vessel image was minimized with this concentration. Air alone as contrast medium gave satisfactory definition of the major arteries. The double-contrast method proved very satisfactory, and the images obtained were of very high quality with high resolution and contrast.

Because the arteries were all normal, it was difficult to evaluate whether the information content varied with different degrees of magnification.

\section{EXPERIMENTS ON SPECIMENS}

\section{Material and Methods}

Two $7 \mathrm{~cm}$ long specimens of the superficial femoral artery were obtained from two cadavers. One was from a 90 year-old woman with numerous partially calcified arteriosclerotic changes in the wall. The other was from a 25 -year-old woman with a normal artery. These two artery specimens were filled with various types of contrast material and placed centrally in an $11 \mathrm{~cm}$ thick plastic container filled with water. The $11 \mathrm{~cm}$ thickness reproduces the scatter and absorption generated by the thigh of an ordinary supine patient.

The same radiographic technique was used as in the canine experiments. Subjective comparison between the amount of information in images obtained at various magnification was performed.

Images were obtained of the arteries filled with air only. Double contrast was then created by coating the inner surface of the artery with Dionosil, leaving the arteries otherwise filled with air. Finally, the arteries were filled with Dionosil (iodine content $270 \mathrm{mg} / \mathrm{ml}$ ) and Dionosil diluted to $135 \mathrm{mgl} / \mathrm{ml}$. Dionosil was chosen because it is almost non-diffusible and permits repeated experiments on the same specimen.

\section{Results}

As in the dog experiments, $70 \mathrm{kV}$ proved to offer maximum-definition angiograms. Angiograms were taken at 1.2, 3,6 and 10 magnifications. With the $6 \times$ magnification, minor detail, particularly the serrate appearance of the slightly contracted normal femoral artery, could be clearly demonstrated, whereas this was not possible with the $3 \times$ magnification when other factors were equal (Fig. 3). Magnification higher than $6 \times$ gave no additional information.

Air contrast, double contrast and complete filling of the lumen of the arteries with Dionosil were all satisfactory for achieving good-detail images of the arteries and their walls. However, the density of undiluted Dionosil was too high to permit penetra- 

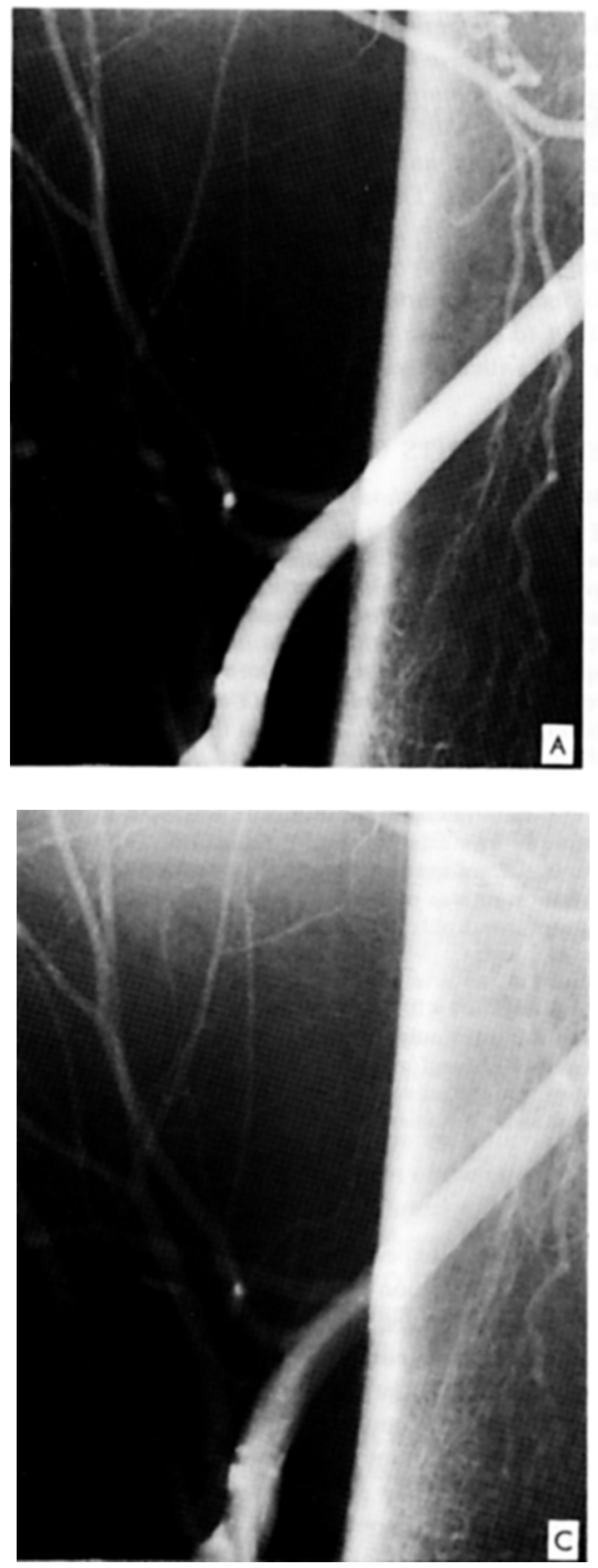

Fig. 2. Effect of variation of iodine concentration of contrast medium in femoral arteriography in the dog. (A) 145 $\mathrm{mgI} / \mathrm{ml}$. (B) $120 \mathrm{mgl} / \mathrm{ml}$. (C) $100 \mathrm{mgl} / \mathrm{ml}$. 6x magnification, $70 \mathrm{kV}, 30 \mathrm{~mA}, 0.6 \mathrm{sec} 0.1 \mathrm{~mm}$ focal spot. Injection rate and amount of contrast agent were the same in all three angiograms.

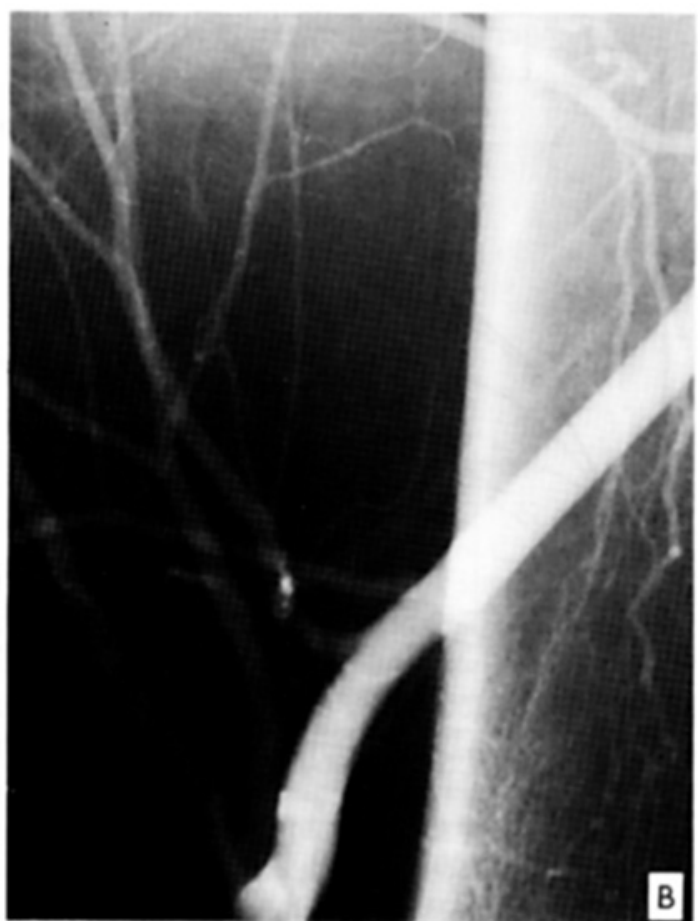

tion in the center of these large arteries. The best definition was obtained with double contrast.

The rare-earth screens combined with Cronex 4 film proved to be superior to the High-Plus Cronex 4 film-screen combination.

\section{DISCUSSION}

These experiments show that $6 \times$ magnification with a $0.1 \mathrm{~mm}$ focal spot can show detail in the walls of major arteries that cannot be identified with standard radiography or even $3 \times$ magnification. It is desirable to be able to achieve this degree of enlargement when studying the femoral artery in patients. In many angiographic rooms this degree of magnification can be obtained by raising the tabletop to a high position and placing the cassette on the floor. This would permit the patient to remain supine during the study and thus greatly facilitate the reproducibility of the imaging from one occasion to another.

The rare-earth screen in combination with Cronex 4 film seems to be the most suitable combination for this type of study. They represent high resolution and a speed increase by a factor of about 1.8 greater than the High-Plus Cronex 4 film-screen combination. Substitution of Cronex 4 with greensensitive Agfa Gevaert Curix Ortho film can pro- 

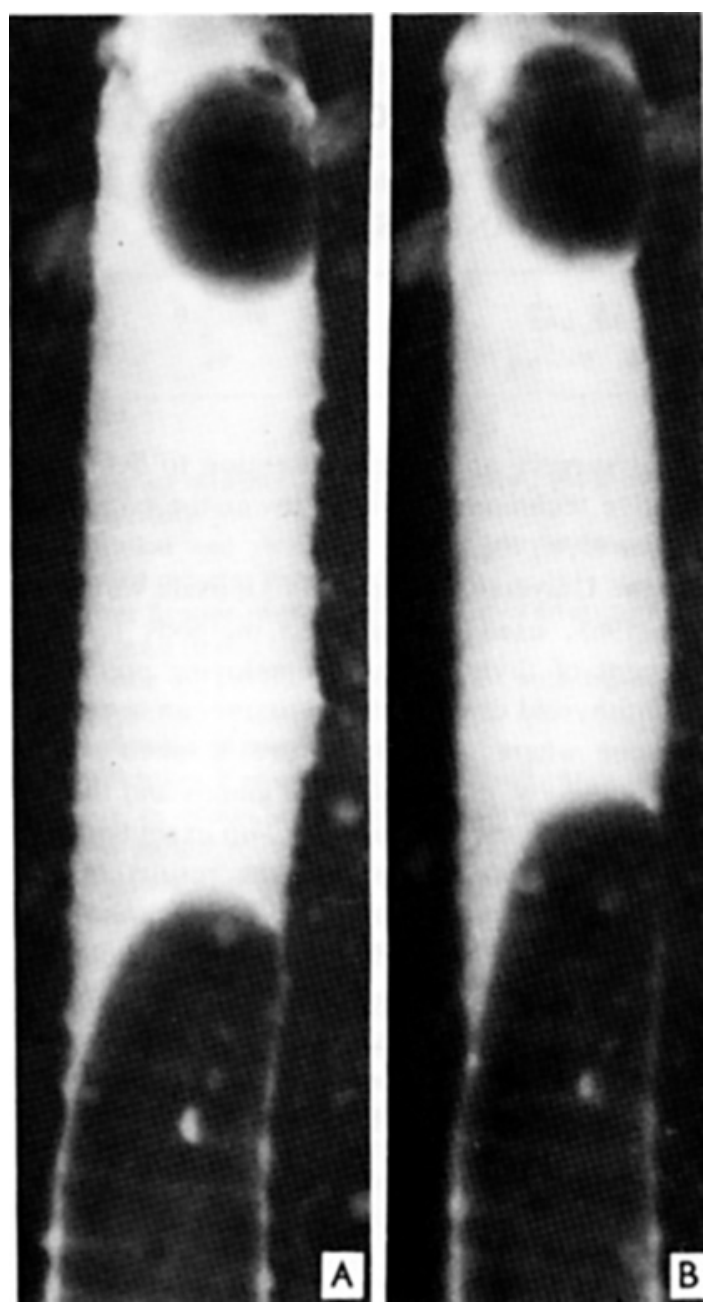

Fig. 3. Normal human femoral artery specimen partially filled with Dionosil. (A) $\times 6$. (B) $\times 3$. Other factors being equal: $0.1 \mathrm{~mm}$ focal spot, $70 \mathrm{kV}, 30 \mathrm{~mA}$. Better detail visualization with $6 \times$ magnification, particularly of serrated appearance of the wall of the artery.

vide a further speed increase by about $50 \%$. This type of angiography requires single exposures only and involves no risk of overheating the tube with a microfocal spot.

Diluted contrast medium with an iodine content of approximately $120 \mathrm{mgI} / \mathrm{ml}$ is probably best for demonstrating both small vessels and details in the walls of large arteries. This is in agreement with previous determinations of the iodine concentration required to achieve good angiographic results (2). This could be achieved by injecting a fairly large amount of diluted contrast medium during a period ' long enough to cover the relatively long exposure necessary. It is expected that these injections of diluted contrast media would be well tolerated by patients.

Other modifications of the angiographic techniques such as use of carbon dioxide or oxygen alone or in combination with iodine-containing contrast agents to create a double-contrast effect, are more difficult to use in patients because these techniques probably will require the occlusion of the artery with a balloon or a similar device. While air alone as contrast gave satisfactory definition of the major arteries it is questionable whether small changes in the walls were in fact detected with this method. It may require the use of $\mathrm{X}$-rays generated at much higher $\mathrm{kV}$ to take full advantage of air as contrast for large vessel angiography.

From the above it seems that $6 \times$ magnification arteriography of major arteries using a $0.1 \mathrm{~mm}$ focal spot would be a feasible and reproducible method in most patients. The risks involved would most likely be no greater than with other angiographic studies. The additional injection of $20-30 \mathrm{ml}$ of diluted water-soluble contrast medium in the iliac or femoral artery will hopefully be well tolerated.

\section{ACKNOWLEDGEMENT}

This work was supported by USPHS grants GM18674 and HL11668.

\section{REFERENCES}

1. Sandor, T., Adams, D. F., Herman, P. G., Eisenberg, H. \& Abrams, H. L.: The potential of magnification angiography. Am J Roentgenol 120:916-921, 1974.

2. Swart, B. \& Dingendorf, W.: Experimenteller Beitrag zur optimalen Gefassdarstellung. Fortschr Rontgenstr 97: 637-648, 1962.

Received June 15, 1974

Address for reprints:

Lars Björk, M.D.

Department of Radiology

Harvard Medical School

25 Shattuck Street

Boston, Massachusetts 02115

USA 